\title{
Central Nervous System Reorganization in a Variety of Chronic Pain States: A Review
}

\author{
Douglas E. Henry, MD, Anthony E. Chiodo, MD, Weibin Yang, MD
}

\begin{abstract}
Chronic pain can develop from numerous conditions and is one of the most widespread and disabling health problems today. Unfortunately, the pathophysiology of chronic pain in most of these conditions, along with consistently effective treatments, remain elusive. However, recent advances in neuroimaging and neurophysiology are rapidly expanding our understanding of these pain syndromes. It is now clear that substantial functional and structural changes, or plasticity, in the central nervous system (CNS) are associated with many chronic pain syndromes. A group of cortical and subcortical brain regions, often referred to as the "pain matrix," often show abnormalities on functional imaging studies in persons with chronic pain, even with different pain locations and etiologies. Changes in the motor and sensory homunculus also are seen. Some of these CNS changes return to a normal state with resolution of the pain. It is hoped that this knowledge will lead to more effective treatments or even new preventative measures. The purpose of this article is to review recent advances in the understanding of the CNS changes associated with chronic pain in a number of clinical entities encountered in the field of physical medicine and rehabilitation. These clinical entities include nonspecific low back pain, fibromyalgia, complex regional pain syndrome, postamputation phantom pain, and chronic pain after spinal cord injury.
\end{abstract}

PM R 2011;3:1116-1125

\section{INTRODUCTION}

Central nervous system (CNS) plasticity refers to the ability of the CNS to reorganize over time. CNS plasticity occurs normally throughout life in response to a person's experiences, thoughts, and actions. The main mechanisms include functional changes or alterations in the intrinsic properties of the neurons and structural mechanisms, which refer to changes in the number or location of synapses between neurons [1]. Although plasticity is best understood in the context of motor skill acquisition and recovery from CNS injury, it is becoming clear that reorganization also occurs in chronic pain states.

\section{BRAIN ACTIVITY IN PERSONS WITH ACUTE PAIN}

It is important to understand the relative activity of specific brain regions in healthy persons who are in acute pain to understand changes that are specific to chronic pain. A metaanalysis that evaluated 152 studies of acute and chronic pain which used a variety of hemodynamic, neuroelectrical, and neurochemical methods determined that several brain regions, sometimes referred to as the "pain matrix," were consistently activated during pain perception [2]. The pain matrix generally is thought to include the primary (S1) and secondary (S2) somatosensory cortex, insula, anterior cingulate cortex (ACC), amygdala, prefrontal cortex (PFC), and thalamus [2]. The study also demonstrated some differences between acute and chronic pain, the most prominent difference being that chronic pain more often activates brain regions involved in cognitive and/or emotional pain processing. Activation of the insula, S1, S2, and lateral thalamus are thought to be related to the sensory-discriminative aspects of pain processing [3], whereas the ACC appears to participate in affective and attentional concomitants of pain sensation, or perceiving pain as an unpleasant experience [4-7]. Foltz and White [8] showed that, after cingulotomy, the
D.E.H. Department of Developmental and Rehabilitative Pediatrics, Children's Hospital, Cleveland Clinic, 2801 MLK Jr Dr, Cleveland, $\mathrm{OH}$ 44104. Address correspondence to: D.E.H.; e-mail: henryd@ccf.org Disclosure: nothing to disclose

A.E.C. Department of Physical Medicine and Rehabilitation, University of Michigan, Ann Arbor, MI

Disclosure: nothing to disclose

W.Y. VA North Texas Health Care System, University of Texas Southwestern Medical School, Rehabilitation Institute of Texas, Dallas, TX

Disclosure: nothing to disclose

Disclosure Key can be found on the Table of Contents and at www.pmrjournal.org

Submitted for publication February 3, 2011; accepted May 22, 2011 
subjects will feel pain but are not bothered by it. Persons with more pain sensitivity and greater levels of perceived unpleasantness to painful stimulation demonstrate increased ACC activity [9].

Posterior parietal and prefrontal cortices are thought to be involved in attentional, cognitive, and memory networks activated by noxious stimuli [3,7]. Attentional modulation of sensory processing and of pain processing share general mechanisms. However, there seem to be additional specific processes or pathways that allow attention to exert even more modulation of pain. The descending pain modulation system includes the PFC and ACC and exerts its influence on the periaqueductal gray matter and posterior thalamus $[10,11]$.

\section{CNS ACTIVITY IN PERSONS WITH CHRONIC PAIN}

The theory of the pain matrix is an extension of the gate control theory of Melzack and Wall [12]. This theory purports that changes in the pain matrix after injury can result in central sensitization. Purported centralization mechanisms include decreased threshold of nociceptor afferent peripheral terminals, degeneration of C-fiber terminals in lamina II, ectopic foci in the dorsal root ganglion, and induced sprouting of A-fibers. Decreased $\boldsymbol{\gamma}$-aminobutyric acid (GABA) receptor activity, downregulation of opioid receptors, and sympathetic ephaptic transmission with nociceptor activation would result. Inflammatory mediators in the periphery can include histamines, purines, leukotrienes, norepinephrine, cytokines, nerve growth factor, neuropeptides, serotonin, prostaglandins, and bradykinin.

Centrally, an activity-dependent increase in spinal neuron excitability occurs with denervation hypersensitivity and synaptic structural changes mediated by N-methyl-D-aspartate receptors and glutamate, along with death of inhibitory neurons, replacement with new afferent excitatory neurons, and establishment of aberrant excitatory synaptic connections [12].

Studies of brain changes in the chronic pain state are necessarily much different than studies of brain changes in the acute pain state. It would be impractical in most situations to image the brain in the healthy, nonpainful state and then compare the changes before and after the onset of pain. Furthermore, these CNS changes are likely influenced by comorbidities, such as depression, anxiety, medication use, decreased physical activity, and limited social stimulation; so it is not surprising that current studies demonstrate a greater variety of CNS changes in chronic pain syndromes than in acute pain states. Nevertheless, some findings are common across the various diagnostic entities and across different imaging modalities. For example, reorganization in the primary motor and sensory homunculus maps occurs in persons with complex regional pain syndrome (CRPS), phantom pain, and back pain. In persons with CRPS, the S1 represen- tation of the affected arm is smaller and shifted in location on the homunculus toward the lip region $[13,14]$. However, in some conditions, such as chronic back pain, the S1 representation in the affected area is larger [13].

In volumetric magnetic resonance imaging (MRI) studies, although the specific decrease in areas of gray matter varies somewhat with different diagnoses, the cingulate cortex, insula, and dorsolateral prefrontal cortex (DLPFC) usually show atrophy across the diagnoses $[15,16]$. Results of other studies suggest a decrease in gray matter in areas involved in pain processing in patients with chronic pain [17]. Results of many of these studies find a correlation between brain gray matter changes and the duration or intensity of pain $[18,19]$. As an example, Rodriguez-Raecke et al [15] studied 32 subjects with chronic pain due to primary hip osteoarthritis by using 3 Tesla MRI and found decreased gray matter in the ACC, right insular cortex and operculum, DLPFC, amygdala, and brainstem compared with control subjects. In a subgroup of 10 patients who were completely pain free after total hip replacement surgery, gray matter increases were found in the ACC, DLPFC, amygdala, and brainstem at 6 weeks and 4 months after surgery.

Functional imaging studies in persons with chronic pain have led to a much greater understanding of how pain is processed in the CNS. For example, in several imaging studies, distraction from pain is associated with decreased painrelated activations in pain matrix regions $[11,20]$. In addition, attention to pain is associated with an increased functional coupling in many of those regions [21,22], which may begin to explain how treatments such as cognitive behavioral therapy, relaxation techniques, and virtual reality [23] benefit patients with chronic pain. Chronic pain states may lead to the development of pain memories that alter the somatotopic map in S1 and may contribute to a hyperalgesic state even without peripheral nociceptive stimulation. These pain memories can be influenced by psychological interventions such as operant and classical conditioning [24,25]. Interestingly, abnormal CNS responses to painful stimuli may develop from as far back as the neonatal period [26]. A group of school-age children who required painful procedures as neonates and a control group with an uncomplicated postnatal course underwent functional MRI (fMRI) during moderately painful heat stimuli. The "procedure" group demonstrated higher activations than did control subjects in most of the pain matrix areas. Furthermore, their pain ratings showed a tendency for increased sensitization and a lack of habituation across trials.

The descending pain modulating system can inhibit or facilitate peripheral nociceptive input. This system receives input from the prefrontal, cingulate, and anterior insular cortices, as well as the amygdala, which allows influence from affective and cognitive processes [27]. Although long-term nociceptive input is known to result in augmented descending inhibition, this enhanced inhibition may amplify neuro- 
nal sensitization in the dorsal horn, thereby promoting a chronic pain state [28].

Predisposing intrinsic factors influence the development of chronic pain and the associated neural plasticity. Although it is likely that many factors are involved, these factors may include individual differences in the ability of the CNS to reorganize. Evidence indicates that individual differences in endogenous pain modulation are risk factors for the development of chronic pain [29,30]. Genotype brain-derived neurotrophic factor polymorphism (present in 30\% of the population) reduces motor map reorganization and corticospinal excitability after training on several fine motor tasks compared with subjects without the polymorphism [31]. A genetic predisposition exists in persons with CRPS [32] and fibromyalgia syndrome (FMS) [29]. Thus genotype clearly plays some role in the development of chronic pain.

\section{CHRONIC BACK PAIN}

Chronic back pain (CBP) is a common medical condition. The study of brain plasticity in persons with CBP is relatively new. Flor et al [33] studied 10 subjects with low back pain and 9 matched healthy control subjects and found evidence of functional reorganization of S1 in the subjects with CBP. They also found that the amount of reorganizational change increased with the chronicity of pain.

Apkarian et al [16] presented the first study that showed brain morphometric (structural) changes in persons with CBP. They compared brain morphology of 26 patients with CBP to 26 matched control subjects by using a 1.5 Tesla Tl-weighted MRI scanner. The study showed a $5.4 \%$ decrease in gray matter volume in the subjects with CBP compared with normal control subjects. When the volumes were compared again after correcting for age, gender, and pain duration, the resultant gray matter volume decrease reached $11 \%$ in subjects with CBP, which is equivalent to the loss of neocortical gray matter volume with $10-20$ years of normal aging. The study further demonstrated that the mean gray matter volume was not different between neuropathic and non-neuropathic subtypes. However, in patients with neuropathic CBP, pain duration was positively correlated with gray matter decrease of $1.3 \mathrm{~cm}^{3}$ per year of chronic pain. Although the study by Apkarian et al [16] indicated that gray matter density was reduced in the bilateral DLPFC and the right anterior thalamus, Schmidt-Wilcke et al [34] found a significant decrease in gray matter in the brainstem and somatosensory cortex. They also found a significant increase in gray matter bilaterally in the basal ganglia and left thalamus.

Baliki et al [35] studied fMRI changes associated with fluctuating phases of CBP $(\mathrm{N}=13)$. When the subjects had a sustained high level of pain, brain mapping showed activity in a single cluster within the medial prefrontal cortex (mPFC) in and around the rostral ACC (rACC), which extended to the level of the genu. Additional analysis showed increased ac- tivity in the posterior thalamus, ventral striatum, and extended amygdala, as well as a larger activity spread within the PFC, but did not reveal any activity in the parietal and insular regions. When the subjects had increasing pain, the brain mapping showed activity in the right anterior and posterior insula, multiple portions of mid ACC, S2, the foot region of $\mathrm{S} 1$, and the cerebellum. It was concluded that, when back pain is high and sustained, it engages brain areas involved in emotion, cognition, and motivation. In contrast, the increasing phase of CBP transiently activated brain regions commonly observed for acute pain.

A possible relationship between $\mathrm{CBP}$ and the so called Default-Mode Network (DMN) was studied by Baliki et al $(\mathrm{n}=30)$ [36]. The DMN is the most active brain system when the brain is doing mental exploration at wakeful rest, without external disturbance [37]. In the study by Baliki et al [36], the subjects with CBP displayed reduced deactivation in several key DMN regions, such as the mPFC, amygdala, and posterior cingulate cortex. The extent of $\mathrm{mPFC}$ deactivation correlated with the number of years of pain. A recent study $(n=8)$ [38] demonstrated that use of a lidocaine patch not only decreased CBP but also reversed the brain plasticity caused by CBP. In this study, fMRI showed a significant decrease in brain activity in the MPFC, rACC, superior frontal gyrus, and posterior parietal cortex after administration of lidocaine.

In summary, results of studies have demonstrated that CBP is associated with decreased gray matter density of the bilateral DLPFC, right anterior thalamus, brainstem, and somatosensory cortex, and increased gray matter bilaterally in the basal ganglia and left thalamus. A sustained high level of back pain increases $\mathrm{MPFC}$ activity, which participates in the emotional, cognitive, and motivational processing of pain. Increasing back pain activates a separate, specific set of brain areas for acute pain. Results of one small study suggest that brain plasticity caused by CBP can be reversed if the pain is alleviated.

\section{FIBROMYALGIA}

FMS is a pain-amplification syndrome. Persons with FMS have increased sensitivity to painful and nonpainful stimuli, including touch, heat, cold, light, sound, and smell [39]. The criteria and scope of fibromyalgia are under considerable debate. In fact, it has been proposed that fibromyalgia is part of a much larger continuum of somatic complaints, multiple sensory hypersensitivities, and low pain thresholds, including chronic fatigue syndrome, irritable bowel syndrome, temporomandibular joint disorder, chronic pelvic pain, nonspecific back pain, and others, and that together, these should be termed central sensitivity syndromes [29]. This proposal has been made in part because of an increasing understanding that abnormalities of central pain processing are an important part of this collection of disorders [40]. 
Results of many studies demonstrate the existence of hyperalgesia and/or allodynia in otherwise nonpainful areas in a number of these conditions, including idiopathic low back pain, irritable bowel syndrome [41], temporomandibular joint disorder [42], vulvodynia [43], and tension headaches $[44,45]$. Results of other studies suggest centrally mediated pain augmentation in persons with fibromyalgia [29], low back pain [46], and irritable bowel syndrome $[47,48]$. One element of this central pain augmentation is attenuated diffuse noxious inhibitory control (DNIC). DNIC refers to the recruitment of descending analgesic pathways to increase an individual's pain threshold after an acute painful stimulus is given [49]. A deficiency in DNIC is known to be present in persons with fibromyalgia [50] and temporomandibular joint disorder [51]. Patients with fibromyalgia also are known to have a low noxious threshold for auditory tones and to tactile pressure $[52,53]$, which suggests a biologic amplification of sensory stimuli. This suggestion is supported by the fact that the insula is commonly overactive in imaging studies in patients with fibromyalgia. The insula appears to play an important role in sensory integration, with the anterior insula specifically involved in the emotional processing of sensations [54,55]. Gracely et al [56] studied 16 fibromyalgia subjects and 16 control subjects with fMRI. When pressure was applied to the left thumb adequate to cause equivalent pain in the 2 groups (less pressure was needed in the fibromyalgia group), there were mostly similar findings. Both groups showed increased metabolic activity in areas associated with sensory discriminative processing (contralateral S1 and S2), motor association (contralateral putamen and ipsilateral cerebellum), sensory association (contralateral superior temporal gyrus and inferior parietal lobule), and affective processing (contralateral insula). However, when the same low-intensity pressure was applied to both groups (which caused pain in the subjects with fibromyalgia but not in the control subjects) fMRI findings were similar to those previously mentioned in the subjects with fibromyalgia, but the control subjects only demonstrated increased activity in the ipsilateral medial frontal gyrus [56]. When painful heat stimuli were used in an otherwise similar study, findings similar to the study by Gracely et al [56] were observed when the stimuli were subjectively similar between the groups. When the stimuli were the same temperature, the fibromyalgia group showed increased activation in the contralateral insular cortex. The placebo group showed increased activity only in a portion of the parietal cortex and mid insular cortex.

A study that used the serotonin norepinephrine reuptake inhibitor milnacipran showed CNS changes in association with clinical improvement [57]. Ninety-two female subjects with fibromyalgia participated in a 13-week, double-blind, placebo-controlled, randomized trial to assess the effects of $100 \mathrm{mg}$ of milnacipran taken twice daily. Preliminary fMRI results showed activations typically seen in persons with fibromyalgia (S1, S2, insula, cingulate cortex, cerebellum, thalamus, and amygdala). At 12 weeks, the group that received milnacipran showed a reduction in Visual Analog Scale ratings compared with control subjects that approached significance and reached significance in subgroups. On fMRI, the group that received milnacipran exhibited increased activation in the amygdala, caudate nucleus, and anterior insula, all of which are areas thought to be involved in the DNIC [55].

Results of other fMRI studies arrived at different results. By using MRI voxel-based morphometry, Hsu et al [58] found no imaging differences in subjects with fibromyalgia compared with healthy individuals when controlling for affective disorder took place, and in subjects with affective disorder, abnormalities were only seen in the left anterior insula. Results of several FMS studies show a reduced regional cerebral blood flow (rCBF) in one or both thalami $[59,60]$. The reason for this finding is unknown, but it is "consistent with a tonic inhibition in response to a persistent excitatory input associated with widespread chronic pain, which is characteristic of FMS" [61]." Guedj et al [62] used single-photon emission computed tomography (SPECT) to evaluate $\mathrm{rCBF}$ in the midbrain in subjects with fibromyalgia. They were treated with subcutaneous ketamine in increasing doses for 10 days (maximum dose, $100 \mathrm{mg}$ ). Eleven of the 17 subjects were considered responders (ie, they had a more than 50\% decrease in the Visual Analog Scale; on average, 82 $\mathrm{mm}$ decreased to $32 \mathrm{~mm}$ ). The postketamine SPECT showed a greater increase in mid brain $\mathrm{rCBF}$ in the responders compared with the nonresponders, which suggests a blockade of the excitatory descending modulation of pain with ketamine. Another SPECT study revealed diminished resting rCBF in bilateral thalami and caudate nuclei in patients with fibromyalgia compared with control subjects [59,63]. Neuroimaging in persons with FMS is extensively reviewed by Nebel and Gracely [57].

\section{CRPS}

CRPS usually develops after a sprain or fracture in a distal limb. It is manifested by sensory, motor, and autonomic symptoms [64]. The pathogenesis is unknown. Evidence exists for peripheral neurogenic inflammation, endothelial dysfunction, pathologic sympathetico-afferent coupling, and CNS changes [28]. Whatever the initiating pathophysiology is, there is an increasing understanding of the structural and functional changes that occur in the CNS of persons affected by CRPS.

The first functional imaging study that showed CNS changes in persons with CRPS was carried out by Fukumoto et al [65]. They used SPECT to demonstrate an alteration in contralateral thalamic perfusion in persons with CRPS. Maihofner et al $[14,66,67]$ performed a series of enlightening experiments on subjects with upper-extremity CRPS, which demonstrate not only that functional cortical changes occur 
in this disorder but that these changes reverse with resolution of symptoms. These investigators used magnetoencephalography to show that the cortical representation of the contralateral (affected) hand was smaller and shifted toward the lip region. The extent of this cortical reorganization was correlated with the severity of pain and mechanical hyperalgesia on the CRPS limb [14]. Some of these same subjects, who had much reduced pain upon being tested more than a year later, showed a normalization of the previously noted cortical changes [66]. The same investigators later studied changes in the motor cortex by using fMRI in 10 subjects with CRPS and with an average duration of symptoms of 52 weeks [67]. During finger tapping movements on the affected side, subjects with upper-extremity CRPS showed markedly increased activation of the contralateral primary and supplementary motor cortices compared with control subjects. They also showed a markedly increased activation in the ipsilateral motor cortex. In addition, activations in the postparietal, supplemental motor area and primary motor cortex were correlated with the degree of motor impairment. In that same study, the investigators had subjects undergo a reaching task and, compared with control subjects, the patients with CRPS showed a prolonged reach pattern consistent with a disturbed integration of visual and proprioceptive inputs in the post parietal cortex. This finding, along with the fMRI data, implied a dysfunction of the posterior parietal and supplemental motor cortices in persons with CRPS. A smaller S1 representation in CRPS also is seen in other studies that used fMRI [68], somatosensory evoked potential mapping [69], and magnetoencephalography [70,71]. Pleger et al [68] showed reduced cortical signals on fMRI in both S1 and S2 as well as impairment of tactile perception on the CRPS side in subjects with CRPS versus control subjects.

Reversal of some CNS changes in persons with CRPS with improvement in symptoms also has been shown when using fMRI $[72,73]$. A study of children with lower-extremity CRPS demonstrated abnormal activation in the anterior and middle ACC, anterior and posterior insula, thalamus, and basal ganglia [73]. When spontaneous pain had resolved and pain to tactile stimuli was absent or minimal, some changes in activation persisted. As in adult studies [74], prominent activations were found in the temporal lobes, perhaps associated with fear and anxiety associated with pain [75]. Changes also were seen in the hypothalamus, which, in turn, may begin to explain the autonomic changes seen in the disorder. The researchers noted that, overall, the findings were similar to those in adult studies.

CNS reorganization is a logical explanation for some of the puzzling sensory and motor symptoms seen in persons with CRPS, such as tactile perception changes and dystonia, because these symptoms are seen in persons with other conditions with known CNS changes. Lesions or inactivation in the posterior parietal cortex are known to produce spatial disorientation and misreaching [76]. Results of several studies also suggest impairment of posterior parietal function in persons with CRPS [70]. A motor neglect-like syndrome, sometimes seen in persons with CRPS [77], also is often found in patients with lesions of the parietal cortex [78]. Ribbers et al [79] described motor impairment in affected and unaffected limbs. Moseley [80] found that patients with CRPS took longer to recognize their affected hand in a laterality recognition task. Two functions of the ventromedial prefrontal cortex are emotional decision making [81] and determination of the emotional value of sensory stimuli [82]. Persons with ventromedial prefrontal cortex lesions do poorly on emotional decision-making tasks. Interestingly, persons with CRPS do just as poorly, even when their pain is transiently reduced [83]. Geha et al [84] found gray matter atrophy in the right ventromedial prefrontal cortex, anterior insula, and nucleus accumbens in patients with CRPS. Furthermore, the extent of atrophy was associated with the duration and intensity of pain. By using diffusion tensor imaging, they also found differences that suggested a diffuse reorganization of white matter tracks (altered branching patterns) in persons with CRPS.

\section{PHANTOM LIMB PAIN}

Phantom pain is defined as a painful sensation in the location of an amputated limb. The pain quality typically is described as stabbing, throbbing, burning, or cramping. Its differential diagnosis includes phantom sensation, residual limb sensation, or residual limb pain. Phantom pain is a common phenomenon, occurring in $72 \%$ of amputees within the first week of surgery, with $60 \%$ continuing to experience pain at 6 months. No change in this prevalence occurs during the next 5 years. Factors that correlate to the development of phantom pain include pain that lasts longer than 1 month before amputation, increased postsurgical pain, and psychological factors, including anxiety. The relationship to preamputation pain may be evidence for presurgical pain centralization as a contributing factor to the development of phantom pain [85].

For phantom pain to generate, brain activity increases in areas of the pain matrix, and recruitment of additional corti$\mathrm{cal}$ areas beyond the classic pain matrix, cortical reorganization, and maladaptive neuroplasticity are required, along with changes in neurochemistry, structural brain changes, and disruption of the brain default network. Flor [13] has demonstrated the phenomenon proposed by Melzack [12] Remapping of the somatosensory cortex by using fMRI in response to injury in animal and human studies after amputation has been shown. This reorganization involves extension into the homunculus regions that represented areas lost by neurologic injury by areas in proximity that have maintained neurologic continuity, which is evidence that the cortex is a far more flexible, dynamic, and adaptive system than has been previously appreciated. In the medical litera- 
ture about amputations, this phenomenon is called "referred phantom sensation," in which tactile stimulation of these adjacent areas will result in an experienced phantom sensation. Such findings are not seen in congenital amputees or in young children who have undergone an amputation [86]. Functional MRI shows brain activity changes in the ipsilateral motor cortex [87]. Other areas involved in phantom pain include the thalamus, S1, S2, insula, forebrain, and ACC [27].

Unfortunately, preventive analgesia and preemptive analgesic techniques have shown inconsistent results. In addition, medication and interventional treatments to date have resulted in statistically significant but not clinically significant changes in pain or function [88]. Although deep brain stimulation shows early promise, further study is needed. A small series showed that $50 \%$ of patients improved with deep brain stimulation for phantom pain. It is postulated that this improvement is due to the release of endogenous opioids, activation of ascending pathways from the periaqueductal gray, and activation of the medial dorsal nucleus of the thalamus, which is associated with limbic system activation and connections to the amygdala and cingulate cortex. Activation of an inhibitory thalamocortical-corticofugal pathway with an inhibitory effect of thalamic stimulation upon the lamina I neurons of the dorsal horn occurs, which modulates the pain experience [89].

A systematic review of mirror therapy showed that it was no better than motor imagery in achieving a meaningful, immediate decline in pain. Pain severity improvement was not sustained, and pain medication use, function, and employment were not significantly altered [90].

\section{CHRONIC PAIN AFTER SPINAL CORD INJURY}

Spinal cord injury (SCI) is a very different issue, with damage to ascending and descending spinal cord fibers rather than peripheral nerve injury. This deafferentation, with associated Wallerian degeneration that extends into the cortex, consistently leads to cortical reorganization. An fMRI in patients who are paraplegic showed that intact finger movements evoked increased activation in the primary motor cortex compared with control subjects [91]. This increased activation was seen in areas of the cortex previously represented by the areas that sustained paralysis [92]. Studies also showed activation in the supplementary motor area, parietal cortex, and cerebellum [91].

In animal studies, fMRI showed that deafferented hind limb territories of S1 will respond to stimulation of the unaffected forepaw as early as 3 days after thoracic spinal cord transaction, which indicated how rapidly this reorganization begins. After these early changes, there is a time of relative inactivity, with changes in the thalamus and caudal spinal cord that occurred 6 months after the time of the injury or later. How this time of relative inactivity relates to the time course of pain centralization in human SCI is unknown. Early reorganization may be due to the loss of ascending GABAergic inhibition with release of excitatory influences that could signal cortical sprouting needed to develop extension of activation to areas affected by paralysis [93]. In addition, in further animal experiments, marked downregulation of Nogo receptor messenger ribonucleic acid as early as 1 day after $\mathrm{SCI}$ is seen in areas of the primary somatosensory cortex in which cortical reorganization was observed by fMRI after SCI, while brain-derived neurotrophic factor is upregulated. This finding supports the theory that cortical reorganization after SCI is related to activity of the Nogo signaling system [94]. Neurotrophin-3 augments neuroplasticity in S1, S2, the brainstem, and the thalamus, whereas antibodies to nerve growth factor inhibit reorganization and decrease postinjury hyperalgesia [95].

Animal models also have demonstrated that thalamic neurons upregulate expression of the Nav1.3 sodium channel by 4 weeks after SCI. The Navl.3 sodium channel is a voltagegated sodium channel expressed at high levels during the development of the nervous system. Increased expression of this channel is thought to underlie increased sensitivity to stimulation and to facilitate the neurophysiologic conditions observed in persons with central neuropathic pain [96]

In the SCI literature, evidence of reorganization clearly exists in experimental and human studies when using fMRI. Specifically, precentral gyrus (primary somatosensory) reorganization occurs after SCI. Neuronal regrowth occurs into areas of the cortex that previously served the parts of the body impaired by the SCI. In response to this neuronal regrowth, a decreased 2-point discrimination threshold is seen [97]. Additional human subject clinical and fMRI observations show that stimulation of sensory spared areas on the side of injury will result in phantom sensation in sensation-deficient areas adjacent to it on the same side. An fMRI study confirmed activation of areas of the primary somatosensory cortex previously subserved by the injured areas upon this stimulation [98]. Also, magnetic stimulation can induce pain in these patients.

In a study of 10 American Spinal Injury Association (ASIA) A subjects who had neuropathic pain, 10 ASIA A subjects who did not have neuropathic pain, and 21 control subjects without SCI, fMRI of the somatosensory cortex during light brushing of the right little finger, thumb, and lip showed reorganization in subjects with SCI, with the little finger activation point moving medially toward the region that normally would innervate the legs. The amount of reorganization in subjects with SCI significantly correlated with ongoing pain intensity levels. Furthermore, in subjects with SCI and neuropathic pain, little finger medial displacement was found to correlate positively with the pain intensity [99]. Similarly, in adult subjects with thoracic level AISA A SCI and neuropathic pain, Moore et al [98] demonstrated that sensory 
contact in areas of referred pain on the left or right forearm that projected referred phantom sensations to the ipsilateral chest evoked activity on fMRI in the central postcentral gyrus (the position of the forearm representation) and the medial postcentral gyrus (the area of the chest representation), with more than $1.6 \mathrm{~cm}$ of nonresponsive cortex intervening. In contrast, in areas of the forearm that did not result in referred pain and in neurologically matched patients with SCI who did not have pain, stimulation evoked central but not medial postgyral activation. These findings again support the concept of cortical plasticity expressed in coactivation of nonadjacent representations, which suggests that somatotopic subcortical remapping, projected to the cortex, is the mechanism of perceptual and cortical reorganization after SCI [98].

Gustin et al [100] demonstrated that mental imagery of paralyzed limbs after SCI can result in activation of central processes to evoke pain. In this demonstration, it was shown that pain does not require the activation of peripheral receptors but can be generated by changes in cortical activity initiated by cortical processes.

By using fMRI, movement imagery in subjects with SCI results in significant activation of an atrophic primary motor cortex, rather than the supplementary and cerebellar cortex, as seen in control subjects without SCI. This finding suggests that, after SCI, the motor system undergoes significant functional changes. A significant negative correlation was found between the ongoing pain and the percentage change in fMRI signal intensity [101].

In adults who underwent surgery of symptomatic cervical stenosis with myelopathy, a finger-tapping activity done 6 months after injury showed an increased volume of activation in the left precentral gyrus and postcentral gyrus after surgery. The precentral area was enlarged, whereas the postcentral area diminished compared with control subjects before surgery. This finding is similar to that of other studies, and it may indicate that S1 may require input from the dorsal columns and spinothalamic tracts to maintain cortical volume. The impact on the likelihood of postsurgical recovery is uncertain, although results of other studies indicate a variance between motor and sensory recovery after spinal cord contusion in favor of motor recovery [102]. In a study of 6 patients with varying degrees of SCI, a strong association between the extent of movement-related activation within the primary motor cortex and the return of neurologic function was demonstrated [103].

\section{SUMMARY}

An immense amount of evolving literature demonstrates functional and structural CNS changes in patients with chronic pain. There are some common findings across diagnoses. For example, changes often are seen in the somatosensory map and in the pain matrix regions, which include S1, $\mathrm{S} 2$, the insula, the ACC, the amygdala, the PFC, and the thalamus. More specifically, abnormalities are seen in the insula, S1, S2, and lateral thalamus related to the sensorydiscriminative aspects of pain processing (such as location and texture) and in the ACC related to the affective, or emotional, processing of chronic pain. Many studies find a correlation between brain gray matter changes and the duration or intensity of pain, particularly in the cingulate cortex, insula, and DLPFC. In the few studies that have assessed changes over time, a normalization of the imaging findings generally occurs with improvement in pain. With the possible exception of chronic headache, it does not appear that specific brain regions are dedicated to different pain syndromes. This finding suggests some common maladaptive CNS reaction, or processing of pain, and to some extent supports the grouping of some of these disorders together, as suggested by Ablin and Clauw [29] and Edwards [30]. However, many differences also are seen across diagnostic groups, experimental designs, and temporal factors; although these differences are inconsistent, they raise many questions that need to be answered.

A limitation of current studies is that they do not indicate the direction and sequence of nociceptive processing. However, electrophysiologic studies are beginning to shed light on the temporal sequencing of local activity, and imaging studies will continue to explain more about other aspects of nociceptive processing, such as neurochemical changes, response to medications and placebos, and anticipation of pain. Genetic influences clearly affect the risk of developing and maintaining chronic pain, and some of this influence may reflect differences in brain plasticity mechanisms and potential and response to medications. Ongoing genetic research will certainly add to our knowledge of chronic pain.

Recent functional imaging research has shown that, after a CNS insult such as stroke, various treatments, including active therapy and constraint-induced movement therapy, can beneficially reorganize the CNS and improve functional abilities [1]. As we learn more about CNS processing of acute and chronic pain and behavioral, physical, and pharmacologic interventions, we are uncovering ways to promote beneficial plasticity or to prevent or reverse maladaptive plasticity. For example, by using biofeedback during realtime fMRI, the subjects learned to control the activation of their ACC and were able to reduce their experienced chronic pain [104]. Hypnotic suggestion can induce changes in pain matrix regions in subjects with FMS [105]. Other promising interventions include intensive sensory and/or physical rehabilitation [106], mirror visual feedback [107], motor imagery [80], cognitive behavioral therapy, virtual reality technology [23], and use of the mirror neuron system [108].

\section{CONCLUSION}

As Latremoliere and Woolf noted, "Pain is not then simply a reflection of peripheral inputs or pathology but is also a 
dynamic reflection of central neuronal plasticity [109]." Although the recent surge in knowledge of reorganization and pain processing in the CNS has dramatically increased our understanding of chronic pain, it seems to represent only the tip of the iceberg. Certainly the pace of this research will continue, and we will answer important questions, even as we create many new ones. Because chronic pain is such a widespread and distressing problem, and because the potential to learn more about its underlying pathophysiology and treatment is tremendous, continued funding for related research is certainly a worthwhile investment.

\section{REFERENCES}

1. Johnston MV. Plasticity in the developing brain: Implications for rehabilitation. Dev Disabil Res Rev 2009;15:94-101.

2. May A. Neuroimaging: Visualising the brain in pain. Neurol Sci 2007;28(Suppl 2):S101-S107.

3. Peyron R, Garcia-Larrea L, Gregoire MC, et al. Haemodynamic brain responses to acute pain in humans: Sensory and attentional networks. Brain 1999;122:1765-1780.

4. Rainville P, Duncan GH, Price DD, Carrier B, Bushnell MC. Pain affect encoded in human anterior cingulate but not somatosensory cortex. Science 1997;277:968-971.

5. Zhuo M. Targeting central plasticity: A new direction of finding painkillers. Curr Pharm Des 2005;11:2797-2807.

6. Jones AKPDS. Cortical and thalamic imaging in normal volunteers and patients with chronic pain. In: Besson JM, Guilbaud G, Ollat H, eds. Forebrain Areas Involved in Pain Processing. Paris, France: John Libbey Eurotext; 1995, 229-238.

7. Vogt BA, Sikes RW. The medial pain system, cingulate cortex, and parallel processing of nociceptive information. Prog Brain Res 2000; 122:223-235

8. Foltz EL, White LE. The role of rostral cingulumotomy in "pain" relief. Int J Neurol 1968;6:353-373.

9. Coghill RC, McHaffie JG, Yen YF. Neural correlates of interindividual differences in the subjective experience of pain. Proc Natl Acad Sci U S A 2003; 100:8538-8542

10. Valet $M$, Sprenger $T$, Boecker $H$, et al. Distraction modulates connectivity of the cingulo-frontal cortex and the midbrain during pain: An fMRI analysis. Pain 2004;109:399-408.

11. Wiech K, Ploner M, Tracey I. Neurocognitive aspects of pain perception. Trends Cogn Sci 2008;12:306-313.

12. Melzack R, Wall PD. Pain mechanisms: A new theory. Science 1965; 150:971-979.

13. Flor $\mathrm{H}$, Elbert $\mathrm{T}$, Knecht $\mathrm{S}$, et al. Phantom-limb pain as a perceptual correlate of cortical reorganization following arm amputation. Nature 1995; 375:482-484.

14. Maihofner $C$, Handwerker $H O$, Neundorfer B, Birklein F. Patterns of cortical reorganization in complex regional pain syndrome. Neurology 2003;61:1707-1715.

15. Rodriguez-Raecke R, Niemeier A, Ihle $K$, Ruether W, May A. Brain gray matter decrease in chronic pain is the consequence and not the cause of pain. J Neurosci 2009;29:13746-13750.

16. Apkarian AV, Sosa $Y$, Sonty $S$, et al. Chronic back pain is associated with decreased prefrontal and thalamic gray matter density. J Neurosci 2004;24:10410-10415.

17. May A. Chronic pain may change the structure of the brain. Pain 2008; $137: 7-15$

18. Apkarian AV, Baliki MN, Geha PY. Towards a theory of chronic pain. Prog Neurobiol 2009;87:81-97.
19. Schmidt-Wilcke $T$, Luerding $R$, Weigand $T$, et al. Striatal grey matter increase in patients suffering from fibromyalgia: A voxel-based morphometry study. Pain 2007;132(Suppl 1):S109-S116.

20. Bantick SJ, Wise RG, Ploghaus A, Clare S, Smith SM, Tracey I. Imaging how attention modulates pain in humans using functional MRI. Brain 2002;125:310-319.

21. Hauck M, Lorenz J, Engel AK. Attention to painful stimulation enhances gamma-band activity and synchronization in human sensorimotor cortex. J Neurosci 2007;27:9270-9277.

22. Ohara S, Crone NE, Weiss N, Lenz FA. Analysis of synchrony demonstrates "pain networks" defined by rapidly switching, task-specific, functional connectivity between pain-related cortical structures. Pain 2006;123:244-253

23. Mahrer NE, Gold JI. The use of virtual reality for pain control: $A$ review. Curr Pain Headache Rep 2009;13:100-109.

24. Flor $\mathrm{H}$. Cortical reorganisation and chronic pain: Implications for rehabilitation. J Rehabil Med 2003;(41 Suppl):66-72.

25. Flor $\mathrm{H}$. The modification of cortical reorganization and chronic pain by sensory feedback. Appl Psychophysiol Biofeedback 2002;27:215227.

26. Hohmeister J, Kroll A, Wollgarten-Hadamek I, et al. Cerebral processing of pain in school-aged children with neonatal nociceptive input: An exploratory fMRI study. Pain 2010;150:257-267.

27. Seifert F, Maihofner C. Central mechanisms of experimental and chronic neuropathic pain: Findings from functional imaging studies. Cell Mol Life Sci 2009;66:375-390.

28. Seifert F, Kiefer G, DeCol R, Schmelz M, Maihofner C. Differential endogenous pain modulation in complex-regional pain syndrome. Brain 2009;132:788-800

29. Ablin $\mathrm{K}$, Clauw DJ. From fibrositis to functional somatic syndromes to a bell-shaped curve of pain and sensory sensitivity: Evolution of a clinical construct. Rheum Dis Clin North Am 2009;35:233-251.

30. Edwards RR. Individual differences in endogenous pain modulation as a risk factor for chronic pain. Neurology 2005;65:437-443.

31. Plowman EK, Kleim JA. Motor cortex reorganization across the lifespan. J Commun Disord 2010;43:286-294

32. de Rooij AM, de Mos M, van Hilten JJ, et al. Increased risk of complex regional pain syndrome in siblings of patients? J Pain 2009;10:12501255.

33. Flor $\mathrm{H}$, Braun $\mathrm{C}$, Elbert $\mathrm{T}$, Birbaumer $\mathrm{N}$. Extensive reorganization of primary somatosensory cortex in chronic back pain patients. Neurosci Lett 1997;224:5-8.

34. Schmidt-Wilcke $T$, Leinisch $E$, Ganssbauer $S$, et al. Affective components and intensity of pain correlate with structural differences in gray matter in chronic back pain patients. Pain 2006;125:89-97.

35. Baliki MN, Chialvo DR, Geha PY, et al. Chronic pain and the emotional brain: Specific brain activity associated with spontaneous fluctuations of intensity of chronic back pain. J Neurosci 2006;26:1216512173.

36. Baliki MN, Geha PY, Apkarian AV, Chialvo DR. Beyond feeling: Chronic pain hurts the brain, disrupting the default-mode network dynamics. J Neurosci 2008;28:1398-1403.

37. Buckner RL, Andrews-Hanna JR, Schacter DL. The brain's default network: Anatomy, function, and relevance to disease. Ann N Y Acad Sci 2008;1124:1-38.

38. Baliki MN, Geha PY, Jabakhanji R, Harden N, Schnitzer TJ, Apkarian AV. A preliminary fMRI study of analgesic treatment in chronic back pain and knee osteoarthritis. Mol Pain 2008;4:47.

39. Staud R, Spaeth M. Psychophysical and neurochemical abnormalities of pain processing in fibromyalgia. CNS Spectr 2008;13(Suppl 5):12-17

40. Bennett R. Fibromyalgia: Present to future. Curr Rheumatol Rep 2005;7:371-376 
41. Moshiree B, Price DD, Robinson ME, Gaible R, Verne GN. Thermal and visceral hypersensitivity in irritable bowel syndrome patients with and without fibromyalgia. Clin J Pain 2007;23:323-330.

42. Maixner W, Fillingim R, Booker D, Sigurdsson A. Sensitivity of patients with painful temporomandibular disorders to experimentally evoked pain. Pain 1995;63:341-351.

43. Giesecke J, Reed BD, Haefner HK, Giesecke T, Clauw DJ, Gracely RH. Quantitative sensory testing in vulvodynia patients and increased peripheral pressure pain sensitivity. Obstet Gynecol 2004;104:126133.

44. Langemark M, Jensen K, Jensen TS, Olesen J. Pressure pain thresholds and thermal nociceptive thresholds in chronic tension-type headache. Pain 1989;38:203-210.

45. Gobel H, Weigle L, Kropp P, Soyka D. Pain sensitivity and pain reactivity of pericranial muscles in migraine and tension-type headache. Cephalalgia 1992;12:142-151.

46. Giesecke T, Gracely RH, Grant MA, et al. Evidence of augmented central pain processing in idiopathic chronic low back pain. Arthritis Rheum 2004;50:613-623.

47. Drossman DA. Brain imaging and its implications for studying centrally targeted treatments in irritable bowel syndrome: A primer for gastroenterologists. Gut 2005;54:569-573.

48. Rapps N, van Oudenhove L, Enck P, Aziz Q. Brain imaging of visceral functions in healthy volunteers and IBS patients. J Psychosom Res 2008;64:599-604.

49. Edwards RR, Ness TJ, Weigent DA, Fillingim RB. Individual differences in diffuse noxious inhibitory controls (DNIC): Association with clinical variables. Pain 2003;106:427-437.

50. de Souza JB, Potvin S, Goffaux P, Charest J, Marchand S. The deficit of pain inhibition in fibromyalgia is more pronounced in patients with comorbid depressive symptoms. Clin J Pain 2009;25:123-127.

51. King CD, Wong F, Currie T, Mauderli AP, Fillingim RB, Riley JL III. Deficiency in endogenous modulation of prolonged heat pain in patients with irritable bowel syndrome and temporomandibular disorder. Pain 2009;143:172-178.

52. McDermid AJ, Rollman GB, McCain GA. Generalized hypervigilance in fibromyalgia: Evidence of perceptual amplification. Pain 1996;66: 133-144

53. Geisser ME, Gracely RH, Giesecke T, Petzke FW, Williams DA, Clauw DJ. The association between experimental and clinical pain measures among persons with fibromyalgia and chronic fatigue syndrome. Eur J Pain 2007;11:202-207

54. Craig AD. Human feelings: Why are some more aware than others? Trends Cogn Sci 2004;8:239-241.

55. Tracey I, Mantyh PW. The cerebral signature for pain perception and its modulation. Neuron 2007;55:377-391.

56. Gracely RH, Geisser ME, Giesecke T, et al. Pain catastrophizing and neural responses to pain among persons with fibromyalgia. Brain 2004; $127: 835-843$.

57. Nebel MB, Gracely RH. Neuroimaging of fibromyalgia. Rheum Dis Clin North Am 2009;35:313-327.

58. Hsu MC, Harris RE, Sundgren PC, et al. No consistent difference in gray matter volume between individuals with fibromyalgia and agematched healthy subjects when controlling for affective disorder. Pain 2009;143:262-267.

59. Mountz JM, Bradley LA, Modell JG, et al. Fibromyalgia in women. Abnormalities of regional cerebral blood flow in the thalamus and the caudate nucleus are associated with low pain threshold levels. Arthritis Rheum 1995;38:926-938.

60. Kwiatek R, Barnden L, Tedman R, et al. Regional cerebral blood flow in fibromyalgia: single-photon-emission computed tomography evidence of reduction in the pontine tegmentum and thalami. Arthritis Rheum 2000;43:2823-2833.
61. Mainguy Y. Functional magnetic resonance imagery (fMRI) in fibromyalgia and the response to milnacipran. Hum Psychopharmacol 2009;24(Suppl 1):S19-S23.

62. Guedj E, Cammilleri S, Colavolpe C, de Laforte C, Niboyet J, Mundler $\mathrm{O}$. Follow-up of pain processing recovery after ketamine in hyperalgesic fibromyalgia patients using brain perfusion ECD-SPECT. Eur J Nucl Med Mol Imaging 2007;34:2115-2119.

63. Gracely RH, Petzke F, Wolf JM, Clauw DJ. Functional magnetic resonance imaging evidence of augmented pain processing in fibromyalgia. Arthritis Rheum 2002;46:1333-1343.

64. Stanton-Hicks M, Janig W, Hassenbusch S, Haddox JD, Boas R, Wilson P. Reflex sympathetic dystrophy: Changing concepts and taxonomy. Pain 1995;63:127-133.

65. Fukumoto M, Ushida $T$, Zinchuk VS, Yamamoto H, Yoshida S. Contralateral thalamic perfusion in patients with reflex sympathetic dystrophy syndrome. Lancet 1999;354:1790-1791.

66. Maihofner C, Handwerker HO, Neundorfer B, Birklein F. Cortical reorganization during recovery from complex regional pain syndrome. Neurology 2004;63:693-701.

67. Maihofner C, Baron R, DeCol R, et al. The motor system shows adaptive changes in complex regional pain syndrome. Brain 2007; 130:2671-2687.

68. Pleger B, Ragert P, Schwenkreis P, et al. Patterns of cortical reorganization parallel impaired tactile discrimination and pain intensity in complex regional pain syndrome. Neuroimage 2006;32:503-510.

69. Pleger $B$, Tegenthoff $M$, Schwenkreis $P$, et al. Mean sustained pain levels are linked to hemispherical side-to-side differences of primary somatosensory cortex in the complex regional pain syndrome I. Exp Brain Res 2004;155:115-119.

70. Vartiainen NV, Kirveskari E, Forss N. Central processing of tactile and nociceptive stimuli in complex regional pain syndrome. Clin Neurophysiol 2008;119:2380-2388

71. Juottonen K, Gockel M, Silen T, Hurri H, Hari R, Forss N. Altered central sensorimotor processing in patients with complex regional pain syndrome. Pain 2002;98:315-323

72. Pleger $B$, Tegenthoff $M$, Ragert $P$, et al. Sensorimotor retuning [corrected] in complex regional pain syndrome parallels pain reduction. Ann Neurol 2005;57:425-429.

73. Lebel A, Becerra $L$, Wallin $D$, et al. fMRI reveals distinct CNS processing during symptomatic and recovered complex regional pain syndrome in children. Brain 2008;131:1854-1879.

74. Maihofner C, Neundorfer B, Birklein F, Handwerker HO. Mislocalization of tactile stimulation in patients with complex regional pain syndrome. J Neurol 2006;253:772-779.

75. Charney DS. Neuroanatomical circuits modulating fear and anxiety behaviors. Acta Psychiatr Scand Suppl 2003;(417):38-50.

76. Desmurget M, Epstein CM, Turner RS, Prablanc C, Alexander GE, Grafton ST. Role of the posterior parietal cortex in updating reaching movements to a visual target. Nat Neurosci 1999;2:563-567.

77. Galer BS, Jensen M. Neglect-like symptoms in complex regional pain syndrome: Results of a self-administered survey. J Pain Symptom Manage 1999;18:213-217.

78. Triggs WJ, Gold M, Gerstle G, Adair J, Heilman KM. Motor neglect associated with a discrete parietal lesion. Neurology 1994;44:11641166

79. Ribbers GM, Mulder T, Geurts AC, den Otter RA. Reflex sympathetic dystrophy of the left hand and motor impairments of the unaffected right hand: Impaired central motor processing? Arch Phys Med Rehabil 2002;83:81-85.

80. Moseley GL. Graded motor imagery is effective for long-standing complex regional pain syndrome: A randomised controlled trial. Pain 2004:108:192-198

81. Bechara A, Tranel D, Damasio H. Characterization of the decisionmaking deficit of patients with ventromedial prefrontal cortex lesions. Brain 2000;123:2189-2202. 
82. Kringelbach ML. The human orbitofrontal cortex: Linking reward to hedonic experience. Nat Rev Neurosci 2005;6:691-702.

83. Apkarian AV, Sosa $Y$, Krauss BR, et al. Chronic pain patients are impaired on an emotional decision-making task. Pain 2004;108:129136.

84. Geha PY, Baliki MN, Harden RN, Bauer WR, Parrish TB, Apkarian AV. The brain in chronic CRPS pain: Abnormal gray-white matter interactions in emotional and autonomic regions. Neuron 2008;60:570581.

85. Jensen TS, Krebs B, Nielsen J, Rasmussen P. Immediate and long-term phantom limb pain in amputees: Incidence, clinical characteristics and relationship to pre-amputation limb pain. Pain 1985;21:267-278.

86. Flor H. The parietal lobes. In: Siegel AM, Andersen RA, Freund HJ, Spencer DD, eds. Advances in Neurology, vol 93. Philadelphia, PA: Lippincott, Williams and Wilkins; 2003, 195-204.

87. Schwenkreis P, Pleger B, Cornelius B, et al. Reorganization in the ipsilateral motor cortex of patients with lower limb amputation. Neurosci Lett 2003;349:187-190

88. Wiech K, Kiefer RT, Topfner S, et al. A placebo-controlled randomized crossover trial of the N-methyl-D-aspartic acid receptor antagonist, memantine, in patients with chronic phantom limb pain. Anesth Analg 2004;98:408-413

89. Bittar RG, Kar-Purkayastha I, Owen SL, et al. Deep brain stimulation for pain relief: A meta-analysis. J Clin Neurosci 2005;12:515-519.

90. Moseley GL, Gallace A, Spence C. Is mirror therapy all it is cracked up to be? Current evidence and future directions. Pain 2008;138:7-10.

91. Curt A, Alkadhi H, Crelier GR, Boendermaker SH, Hepp-Reymond MC, Kollias SS. Changes of non-affected upper limb cortical representation in paraplegic patients as assessed by fMRI. Brain 2002;125: 2567-2578.

92. Lotze M, Laubis-Herrmann $U$, Topka H. Combination of TMS and fMRI reveals a specific pattern of reorganization in Ml in patients after complete spinal cord injury. Restor Neurol Neurosci 2006;24:97-107.

93. Endo T, Spenger C, Tominaga T, Brene S, Olson L. Cortical sensory map rearrangement after spinal cord injury: fMRI responses linked to Nogo signalling. Brain 2007;130:2951-2961

94. Endo $T$, Tominaga $T$, Olson L. Cortical changes following spinal cord injury with emphasis on the Nogo signaling system. Neuroscientist 2009; 15:291-299.

95. Ramu J, Bockhorst KH, Grill RJ, Mogatadakala KV, Narayana PA. Cortical reorganization in NT3-treated experimental spinal cord injury: Functional magnetic resonance imaging. Exp Neurol 2007;204: 58-65.
96. Hains BC, Saab CY, Waxman SG. Changes in electrophysiological properties and sodium channel Navl.3 expression in thalamic neurons after spinal cord injury. Brain 2005;128:2359-2371.

97. Song ZK, Cohen MJ, Ament PA, Ho WH, Vulpe M, Schandler SL. Two-point discrimination thresholds in spinal cord injured patients with dysesthetic pain. Paraplegia 1993;31:425-493.

98. Moore CI, Stern CE, Dunbar C, Kostyk SK, Gehi A, Corkin S. Referred phantom sensations and cortical reorganization after spinal cord injury in humans. Proc Natl Acad Sci U S A 2000;97:14703-14708.

99. Wrigley PJ, Press SR, Gustin SM, et al. Neuropathic pain and primary somatosensory cortex reorganization following spinal cord injury. Pain 2009; 141:52-59.

100. Gustin SM, Wrigley PJ, Gandevia SC, Middleton JW, Henderson LA, Siddall PJ. Movement imagery increases pain in people with neuropathic pain following complete thoracic spinal cord injury. Pain 2008; 137:237-244

101. Gustin SM, Wrigley PJ, Henderson LA, Siddall PJ. Brain circuitry underlying pain in response to imagined movement in people with spinal cord injury. Pain 2010;148:438-445.

102. Duggal $N$, Rabin $D$, Bartha $R$, et al. Brain reorganization in patients with spinal cord compression evaluated using fMRI. Neurology 2010; 74:1048-1054

103. Cramer SC, Fray E, Tievsky A, et al. Changes in motor cortex activation after recovery from spinal cord inflammation. Mult Scler 2001;7:364-370.

104. deCharms RC, Maeda F, Glover GH, et al. Control over brain activation and pain learned by using real-time functional MRI. Proc Natl Acad Sci U S A 2005;102:18626-18631

105. Derbyshire SW, Whalley MG, Oakley DA. Fibromyalgia pain and its modulation by hypnotic and non-hypnotic suggestion: An fMRI analysis. Eur J Pain 2009;13:542-550.

106. Sherry DD, Wallace CA, Kelley C, Kidder M, Sapp L. Short- and long-term outcomes of children with complex regional pain syndrome type I treated with exercise therapy. Clin J Pain 1999;15:218223

107. Ramachandran VS, Altschuler EL. The use of visual feedback, in particular mirror visual feedback, in restoring brain function. Brain 2009;132:1693-1710.

108. Ertelt $D$, Small $S$, Solodkin A, et al. Action observation has a positive impact on rehabilitation of motor deficits after stroke. Neuroimage 2007;36(Suppl 2):T164-T173

109. Latremoliere A, Woolf CJ. Central sensitization: A generator of pain hypersensitivity by central neural plasticity. J Pain 2009;10:895-926. 